\title{
Synthesis and antiviral studies of a novel isodideoxynucleoside: an analogue of the antiviral compound, tiazofurin
}

\author{
Vasu Nair* ${ }^{a, b}$ and Thomas Wenzel ${ }^{b}$ \\ ${ }^{a}$ Department of Pharmaceutical and Biomedical Sciences, The University of Georgia, Athens, \\ GA, USA 30602 \\ ${ }^{b}$ Department of Chemistry, University of Iowa, Iowa City, IA, USA 52242 \\ E-mail:vnair@rx.uga.edu
}

(received 23 Jul 04; accepted 23 Sept 04; published on the web 05 Oct 04)

\begin{abstract}
The synthesis and antiviral studies of an isomeric dideoxynucleoside analogue of tiazofurin is described. ${ }^{1} \mathrm{H}$ and ${ }^{13} \mathrm{C}$ NMR studies, including ${ }^{1} \mathrm{H}-\mathrm{NOE}, 2 \mathrm{D}-\mathrm{NOESY}$ and $\left[{ }^{1} \mathrm{H},{ }^{13} \mathrm{C}\right]$ gateddecoupling methods, were used to provide unequivocal evidence for the structure of the target compound.
\end{abstract}

Keywords: Tiazofurin, isodideoxynucleoside, synthesis, NMR, antiviral

\section{Introduction}

The discovery, that tiazofurin (2- $\beta$-D-ribofuranosylthiazole-4-carboxamide, 1 possesses antitumor activity ${ }^{1}$ and is also endowed with broad-spectrum antiviral activity, ${ }^{1,2}$ led to the synthesis of several tiazofurin derivatives, including analogs 2 and $3 .^{3-5}$ In more recent studies, a series of isomeric dideoxynucleosides was synthesized in our laboratory. ${ }^{6-8}$

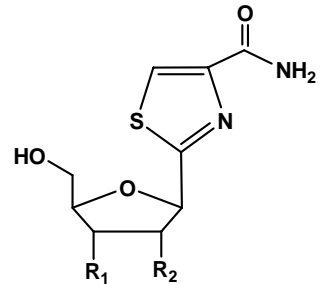

1. $\mathrm{R}_{1}=\mathrm{R}_{2}=\mathrm{OH}$

2. $\mathrm{R}_{1}=\mathrm{OH}, \mathrm{R}_{2}=\mathrm{H}$

3. $\mathrm{R}_{1}=\mathrm{R}_{2}=\mathrm{H}$
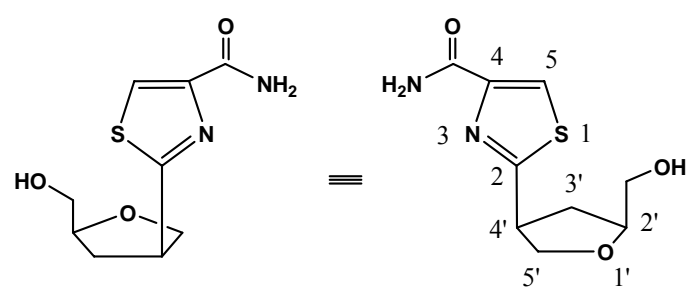

4 
Among them was (-) isodideoxyadenosine, a compound which exhibited potent antiviral activity against HIV-1 and HIV-2. ${ }^{9}$ In our continuing search for new anti-HIV agents, we synthesized and studied the unusual isomeric C-dideoxynucleoside, 4(S)-(4-carbamoyl-thiazol-2yl)-tetrahydro-2(S)-furan-methanol 4 and this paper describes the results of our synthesis and antiviral studies.

\section{Results and Discussion}

Of the several synthetic procedures for obtaining thiazole derivatives, the reaction of thioamides with $\alpha$-halocarbonyl derivatives is the best known. ${ }^{10}$ Thus, the most appropriate starting material in the synthesis of 4 (Scheme 1) was the dideoxyribose $5 .{ }^{8}$ Reaction of 5 with potassium cyanide and [18]-crown-6 in dry DMF for $24 \mathrm{~h}$ at $95^{\circ} \mathrm{C}$ yielded two nitriles 6 (67\%) and its $\alpha$-isomer (5\%). Nitrile 6 was converted into thioamide $7(58 \%)$ by treatment with hydrogen sulfide in the presence of triethylamine for $8 \mathrm{~h}$. Conversion of thioamide 7 into thiazole ester 8 was performed with freshly distilled ethyl bromopyruvate in refluxing ethanol. Surprisingly, two esters, 8 (48\%) and its trans-isomer with the base in the $\alpha$-configuration $(18 \%)$, were formed. The inversion of configuration, while unexpected, may be attributed to an acid-catalyzed isomerization. Treatment of 8 with methanolic ammonia led to the tiazofurin analogue 4 in $79 \%$ yield.

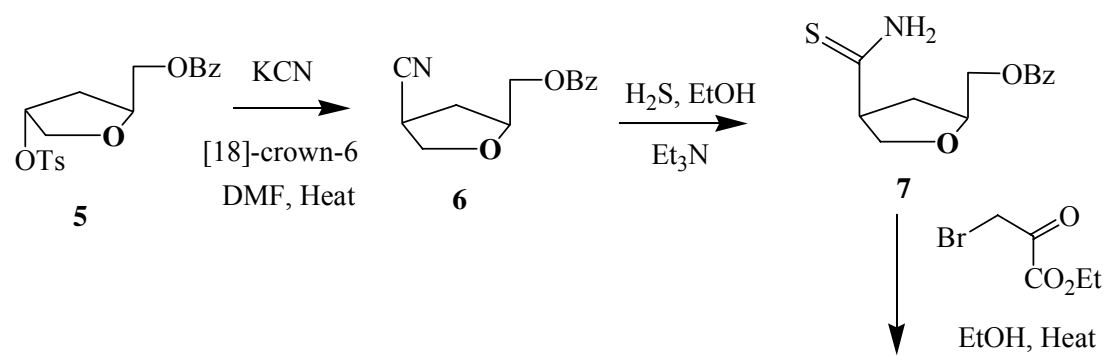

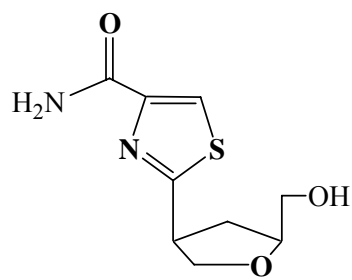

4
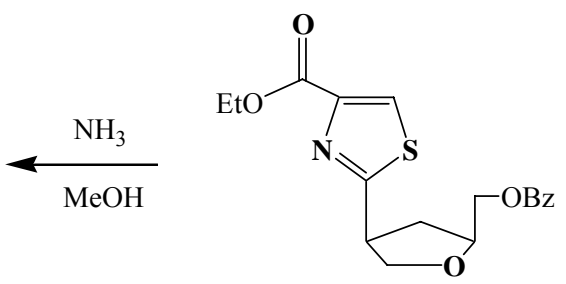

\section{Scheme 1}

Assignment of the configuration at C-4 of compounds 6 and 7 was made on the basis of ${ }^{1} \mathrm{H}$ NOE difference spectroscopy. Upon irradiation of H-4, compounds 6 and 7 showed marked NOE effect on $\mathrm{H}-2 \alpha$, whereas the $\alpha$-isomer did not show enhancement of the resonance of $\mathrm{H}-2$. In addition, for compound 6, irradiation of $\mathrm{H}-2$ showed an NOE enhancement of $3.4 \%$ in $\mathrm{H}-4$, much larger compared to the trans-isomer with the $\alpha$-cyano group. These and other NOE results 
were confirmed by assignments made on the basis of 2D-NOESY NMR data. For example, for the trans-diastereoisomer of $\mathbf{4}$ (with $\alpha$-base), 2D-NOESY NMR spectrum showed a cross-peak between $\mathrm{H}-4$ ' and $-\underline{\mathrm{CH}}_{2} \mathrm{OH}$, whereas this cross-peak was absent from the 2D-NOESY spectrum of 4. Assignment of the ${ }^{13} \mathrm{C}$ data was made on the basis of $\left[{ }^{1} \mathrm{H},{ }^{13} \mathrm{C}\right]$ gated-decoupled NMR spectra.

In vitro anti-HIV studies on the isodideoxytiazofurin 4 in HIV-1 infected cell lines revealed that it was inactive but relatively non-toxic. Antiviral studies against other viruses are planned.

\section{Experimental Section}

General Procedures. Melting points reported are uncorrected and were determined on an Electrothermal Engineering Ltd. Melting point apparatus. Ultraviolet (UV) spectra were recorded on a Cary $3 \mathrm{UV}$-Visible spectrophotometer. ${ }^{1} \mathrm{H}$ NMR and ${ }^{13} \mathrm{C}$ NMR were recorded on a AC-300 and WM-360 instruments. Column chromatographic separations were carried out using 230-400 mesh silica gel. Purities of intermediates and final products were determined by a combination of ${ }^{1} \mathrm{H}$ and ${ }^{13} \mathrm{C}$ NMR spectra, quantitative UV data and chromatographic analysis.

2(S)-(Benzoyloxymethyl)-4(R)-cyano-tetrahydrofuran (6). To a solution of compound 5 (500 $\mathrm{mg}, 1.33 \mathrm{mmol})$ in dry DMF $(10 \mathrm{ml})$ was added potassium cyanide $(135 \mathrm{mg}, 2.07 \mathrm{mmol})$ and [18]-crown-6 (360 mg, $1.36 \mathrm{mmol})$. The solution was stirred at $95^{\circ} \mathrm{C}$ for $24 \mathrm{~h}$ and the solvent was evaporated under reduced pressure. The oily residue was chromatographed on silica gel (column: $20 \times 3 \mathrm{~cm}$, solvent: $\mathrm{CHCl}_{3} /$ EtOAc 9:1). The slower migrating zone yielded $\mathbf{6}$ as colorless oil (190 mg, 62\%). TLC $\left(\mathrm{CHCl}_{3} /\right.$ EtOAc 9:1): $\mathrm{R}_{\mathrm{f}}=0.7 .{ }^{1} \mathrm{H}-\mathrm{NMR}\left(\mathrm{CDCl}_{3}\right): \delta 2.11\left(\mathrm{~m}, 1 \mathrm{H}, \mathrm{H}_{\beta}-3\right) ; 2.50(\mathrm{~m}$, $\left.1 \mathrm{H}, \mathrm{H}_{\alpha}-3\right) ; 3.15$ (m, $\left.1 \mathrm{H}, \mathrm{H}-4\right) ; 4.01$ (dd, $\left.1 \mathrm{H}, \mathrm{J}=7.3,9.0 \mathrm{~Hz}, \mathrm{CH}_{2} \mathrm{OBz}\right) ; 4.10$ (dd, $1 \mathrm{H}, \mathrm{J}=6.1,8.8$ $\mathrm{Hz}, \mathrm{CH}_{2} \mathrm{OBz}$ ); 4.31 (m, 1H, H-2); 4.37 (dd, $\left.1 \mathrm{H}, \mathrm{J}=4.5,11.7 \mathrm{~Hz}, \mathrm{H}_{\alpha}-5\right) ; 4.47$ (dd, $1 \mathrm{H}, \mathrm{J}=3.7$, $\left.11.7 \mathrm{H}_{\beta}-5\right) ; 7.42$ (t, $2 \mathrm{H}, \mathrm{J}=7.7 \mathrm{~Hz}$, arom. $\left.\mathrm{H}\right) ; 7.52$ (t, $1 \mathrm{H}, \mathrm{J}=7.5 \mathrm{~Hz}$, arom. H); 8.04 (d, 2H, J = $7.7 \mathrm{~Hz}$, arom. H). From the faster migrating zone, the $\alpha$-isomer was obtained as a colorless oil (15 mg, 5\%). TLC $\left(\mathrm{CHCl}_{3} /\right.$ EtOAc 9:1): $\mathrm{R}_{\mathrm{f}}=0.75 .{ }^{1} \mathrm{H}-\mathrm{NMR}\left(\mathrm{CDCl}_{3}\right): \delta 2.19\left(\mathrm{~m}, 1 \mathrm{H}, \mathrm{H}_{\beta}-3\right) ; 2.46$ (m, $\left.1 \mathrm{H}, \mathrm{H}_{\alpha}-3\right) ; 3.16(\mathrm{~m}, 1 \mathrm{H}, \mathrm{H}-4) ; 3.94$ (dd, $1 \mathrm{H}, \mathrm{J}=7.0,8.8 \mathrm{~Hz}, \mathrm{CH}_{2} \mathrm{OBz}$ ); 4.03 (dd, 1H, J=7.4, $8.9 \mathrm{~Hz}, \mathrm{CH}_{2} \mathrm{OBz}$ ); $4.21\left(\mathrm{dd}, 1 \mathrm{H}, \mathrm{J}=7.2,11.7 \mathrm{~Hz}, \mathrm{H}_{\beta}-5\right) ; 4.32\left(\mathrm{dd}, 1 \mathrm{H}, \mathrm{J}=5.4,11.7 \mathrm{~Hz}, \mathrm{H}_{\alpha}-5\right) ; 4.46$ (m, 1H, H-2); 7.43 (t, 2H, J=7.8 Hz, arom. H); 7.56 (t, $1 \mathrm{H}, \mathrm{J}=7.5 \mathrm{~Hz}$, arom. H); 8.05 (d, 2H, $\mathrm{J}=7.8 \mathrm{~Hz}$, arom. H). ${ }^{13} \mathrm{C}-\mathrm{NMR}\left(\mathrm{CDCl}_{3}\right): \delta$ 28.6, 32.7, 65.2, 70.3, 77.0, 120.1, 128.4, 129.5, 129.7, 133.2, 166.2.

2(S)-(Benzoyloxymethyl)-4(S)-thiocarbamoyl-tetrahydrofuran (7). A solution of 6 (250 $\mathrm{mg}$, $1.08 \mathrm{mmol})$ in ethanol $(1.8 \mathrm{ml})$ and triethylamine $(200 \mu \mathrm{l})$ was maintained at room temperature while hydrogen sulfide was bubbled into the solution for $6 \mathrm{~h}$. The solvent was removed under reduced pressure and the oily residue was purified by flash chromatography (column: 15 x $3 \mathrm{~cm}$; solvent: $\mathrm{CHCl}_{3} / \mathrm{MeOH}$ 95:5). Isolation of the main zone furnished 7 as a light yellow oil (150 mg, 52\%). TLC $\left(\mathrm{CHCl}_{3} / \mathrm{MeOH} 95: 5\right): \mathrm{R}_{\mathrm{f}}=0.4 .{ }^{1} \mathrm{H}-\mathrm{NMR}\left(\mathrm{CDCl}_{3}\right): \delta 2.16\left(\mathrm{~m}, 1 \mathrm{H}, \mathrm{H}_{\beta}-3\right) ; 2.48(\mathrm{~m}$, 
$\left.1 \mathrm{H}, \mathrm{H}_{\alpha}-3\right) ; 3.53(\mathrm{~m}, 1 \mathrm{H}, \mathrm{H}-4) ; 3.90\left(\mathrm{dd}, 1 \mathrm{H}, \mathrm{J}=6.8,9.2 \mathrm{~Hz}, \mathrm{CH}_{2} \mathrm{OBz}\right) ; 4.13$ (dd, $1 \mathrm{H}, \mathrm{J}=4.8,9.2$ $\left.\mathrm{Hz}, \mathrm{CH}_{2} \mathrm{OBz}\right) ; 4.30$ (m, 1H, H-2); 4.42 (dd, $\left.1 \mathrm{H}, \mathrm{J}=5.9,11.9 \mathrm{~Hz}, \mathrm{H}_{\alpha}-5\right) ; 4.52$ (dd, $1 \mathrm{H}, \mathrm{J}=3.3$, $\left.11.9 \mathrm{H}_{\beta}-5\right) ; 7.44\left(\mathrm{t}, 3 \mathrm{H}, \mathrm{J}=7.8 \mathrm{~Hz}, 2\right.$ arom. $\left.\mathrm{H}, 1 \mathrm{NH}_{2}\right) ; 7.57$ (t, $1 \mathrm{H}, \mathrm{J}=7.6 \mathrm{~Hz}$, arom. H); 7.75 (s, br, $\left.1 \mathrm{H}, \mathrm{NH}_{2}\right) ; 8.03(\mathrm{~d}, 2 \mathrm{H}, \mathrm{J}=8.4 \mathrm{~Hz}$, arom. $\mathrm{H}) .{ }^{13} \mathrm{C}-\mathrm{NMR}\left(\mathrm{CDCl}_{3}\right): \delta 35.4,53.2,65.5,73.1$, $77.5,128.4,129.5,129.7,133.2,166.5,210.5$.

2-[2(S)-(Benzoyloxymethyl)tetrahydrofuran-4(S)-yl]-thiazole-4-carboxylic ethyl ester (8). A mixture of $7(100 \mathrm{mg}, 0.38 \mathrm{mmol})$ and ethyl bromopyruvate $(160 \mu \mathrm{l} 223 \mathrm{mg}, 1.15 \mathrm{mmol})$ in ethanol $(1 \mathrm{ml})$ was refluxed for $6 \mathrm{~h}$ and the solvent was evaporated and the oily residue was purified by flash chromatography (column: $20 \times 3 \mathrm{~cm}$; solvent: $\mathrm{CHCl}_{3} / \mathrm{EtOAc}$ :1). From the slower migrating zone, 8 was isolated as a colorless oil $(65 \mathrm{mg}, 48 \%)$. TLC $\left(\mathrm{CHCl}_{3} / \mathrm{EtOAc} 9: 1\right)$ : $\mathrm{R}_{\mathrm{f}}=0.2 .{ }^{1} \mathrm{H}-\mathrm{NMR}\left(\mathrm{CDCl}_{3}\right): \delta 1.38\left(\mathrm{t}, 3 \mathrm{H}, \mathrm{J}=7.1 \mathrm{~Hz}, \mathrm{CH}_{3}\right) ; 2.11\left(\mathrm{~m}, 1 \mathrm{H}, \mathrm{H}_{\beta}-3^{\prime}\right) ; 2.68(\mathrm{~m}, 1 \mathrm{H}$, $\left.\mathrm{H}_{\alpha^{-}} 3^{\prime}\right) ; 3.98-4.08\left(\mathrm{~m}, 2 \mathrm{H}, \mathrm{CH}_{2} \mathrm{OBz}\right) ; 4.24$ (m, 1H, H-4'); 4.36-4.44 (m, 4H, $\mathrm{H}_{\beta}-5^{\prime}, \mathrm{H}-2^{\prime}$, $\left.\mathrm{OCH}_{2} \mathrm{CH}_{3}\right) ; 4.50\left(\mathrm{~m}, 1 \mathrm{H}, \mathrm{H}_{\alpha}-5^{\prime}\right) ; 7.41$ (t, $2 \mathrm{H}, \mathrm{J}=7.7 \mathrm{~Hz}$, arom. H); 7.54 (t, $1 \mathrm{H}, \mathrm{J}=7.4 \mathrm{~Hz}$, arom. $\mathrm{H}) ; 8.02(\mathrm{~d}, 2 \mathrm{H}, \mathrm{J}=8.3 \mathrm{~Hz}$, arom. $\mathrm{H}) ; 8.05$ (s, $1 \mathrm{H}, \mathrm{H}-5)$. The faster migrating zone ( $\alpha$-isomer) yielded $(25 \mathrm{mg}, 18 \%)$ as slight yellow oil. TLC $\left(\mathrm{CHCl}_{3} / \mathrm{EtOAc}^{9}: 1\right): \mathrm{R}_{\mathrm{f}}=0.25$. ${ }^{1} \mathrm{H}-\mathrm{NMR}$ $\left(\mathrm{CDCl}_{3}\right): \delta 1.37\left(\mathrm{t}, 3 \mathrm{H}, \mathrm{J}=7.1 \mathrm{~Hz}, \mathrm{CH}_{3}\right) ; 2.35\left(\mathrm{~m}, 1 \mathrm{H}, \mathrm{H}_{\beta}-3^{\prime}\right) ; 2.45\left(\mathrm{~m}, 1 \mathrm{H}, \mathrm{H}_{\alpha} \mathbf{3}^{\prime}\right) ; 4.00(\mathrm{~m}, 2 \mathrm{H}$, $\left.\mathrm{CH}_{2} \mathrm{OBz}\right)$; 4.31-4.44 (m, 5H, H-5', H-2', $\left.\mathrm{OCH}_{2} \mathrm{CH}_{3}\right)$; 4.57 (m, 1H, H-4'); 7.42 (t, 2H, J = 7.6 Hz, arom. H); 7.54 (t, $1 \mathrm{H}, \mathrm{J}=7.4 \mathrm{~Hz}$, arom. H); 8.04 (d, 2H, J = 7.9 Hz, arom. H): 8.07 (s, 1H, H-5). ${ }^{13} \mathrm{C}-\mathrm{NMR}\left(\mathrm{CDCl}_{3}\right): \delta 14.3,35.5,43.6,61.5,66.2,73.6,76.5,127.1,128.4,129.6,129.7,133.2$, 146.0, 161.2, 166.4, 172.2.

4(S)-(4-Carbamoyl-thiazol-2-yl)-tetrahydro-2(S)-furanmethanol (4). A solution of compound 8 (100 mg, $0.28 \mathrm{mmol})$ in methanolic ammonia $\left(2 \mathrm{ml}, \mathrm{MeOH}\right.$ saturated with $\mathrm{NH}_{3}$ at $0^{\circ} \mathrm{C}$ ) was stirred at r.t. for $60 \mathrm{~h}$. Then, the solvent was evaporated and the oily residue was purified by $\mathrm{FC}$ (column: $10 \times 3 \mathrm{~cm}$, solvent: $\mathrm{CHCl}_{3} / \mathrm{MeOH}$ 95:5). The main zone was evaporated yielding compound 4 (50 mg, 79\%) as colorless oil. TLC $\left(\mathrm{CHCl}_{3} / \mathrm{MeOH} 95: 5\right): \mathrm{R}_{\mathrm{f}}=0.2 \mathrm{UV}(\mathrm{MeOH})$ : 233 (6900). ${ }^{1} \mathrm{H}-\mathrm{NMR}\left(\mathrm{CDCl}_{3}\right): \delta 2.12\left(\mathrm{~m}, 1 \mathrm{H}, \mathrm{H}_{\beta-3^{\prime}}\right) ; 2.47\left(\mathrm{~m}, 1 \mathrm{H}, \mathrm{H}_{\alpha^{-}} 3^{\prime}\right) ; 2.94$ (s, br, $\left.1 \mathrm{H}, \mathrm{OH}\right)$; 3.64 (m, $\left.1 \mathrm{H}, \mathrm{H}-4^{\prime}\right)$; 3.79-3.87 (m, 2H, $\left.\mathrm{CH}_{2} \mathrm{OH}\right)$; 4.02 (m, $\left.1 \mathrm{H}, \mathrm{H}_{\alpha^{-}} 5^{\prime}\right)$; 4.13-4.20 (m, 2H, $\mathrm{H}_{\beta}-5^{\prime}, \mathrm{H}-$ $\left.2^{\prime}\right) ; 6.24\left(\mathrm{~s}\right.$, br, $\left.1 \mathrm{H}, \mathrm{CONH}_{2}\right) ; 7.16\left(\mathrm{~s}, \mathrm{br}, 1 \mathrm{H}, \mathrm{CONH}_{2}\right) ; 8.02(\mathrm{~s}, 1 \mathrm{H}, \mathrm{H}-5) .{ }^{13} \mathrm{C}-\mathrm{NMR}\left(\mathrm{CDCl}_{3}\right): \delta$ 34.7, 43.5, 64.0, 73.1, 80.4, 123.9, 149.1, 163.0, 171.8. Anal. Calc. for $\mathrm{C}_{9} \mathrm{H}_{12} \mathrm{~N}_{2} \mathrm{O}_{3} \mathrm{~S} 0.75 \mathrm{H}_{2} \mathrm{O}$ : C, 44.71; H, 5.63; N, 11.59. Found: C, 44.92; H, 5.02, N, 11.40

\section{Acknowledgements}

We thank the National Institutes of Health (NIAID) for support of our research. We thank the NCI for the anti-HIV evaluation. 


\section{References}

1. Srivastava, P.C.; Pickering, M.V.; Allen, L.B.; Streeter, D.G.; Campbell, M.T.; Witkowski, J.T.; Sidwell, R.W.; Robins, R.K. J. Med. Chem. 1977, 20, 256.

2. Kirsi, J.J.; North, J.A.; McKernan, P.A.; Murray, B.K.; Canonico, P.G.; Huggins, J.W.; Srivastava, P.C.; Robins, R.K. Antimicrob. Agents Chemother. 1983, 24, 353.

3. Srivastava, P.C.; Robins, R.K., Takusagawa, F.; Berman, H. M.; J. Heterocyclic Chem. 1981, $8,1659$.

4. Upadhya, K.; DaRe, J.; Schubert, E.M.; Chmurny, G. N.; Gabrielson, B. Nucleosides \& Nucleotides 1990, 9, 649.

5. Franchetti, P.; Cappellacci, L.; Grifantini, M.; Barzi, A.; Nocentini, G.; Yang, H.; O’Connor, A.; Jayaram, H. N.; Carrell, C.; Goldstein, B. M. J. Med. Chem. 1995, 38, 3829.

6. Nair, V.; Jahnke, T. S. Antimicrob. Agents Chemother. 1995, 39, 1017.

7. Nair, V. in Recent Advances in Nucleosides: Chemistry and Chemotherapy; Chu, C. K., Ed., Elsevier Science: Amsterdam, Netherlands, 2002, p 149.

8. Nair, V.; Nuesca, Z.M. J. Am. Chem. Soc. 1992, 114, 7951.

9. Nair, V.; St. Clair, M.; Reardon, J.E.; Krasny, H.C.; Hazen, R.J.; Paff, M.T.; Boone, L.R.; Tisdale, M.; Najera, I.; Dornsife, R.E.; Everett, D.R.; Borroto-Esoda, K.; Yale, J.L.; Zimmerman, T.P.; Rideout, J. Antimicrob. Agents Chemother. 1995, 39, 1993.

10. Sprague, J.M.; Land, A.H. In Heterocyclic Compounds, Vol. V, Elderfield, R.C., Ed.; Wiley: New York, N.Y., 1957, p 484. 